\title{
Survival outcomes of low-risk and intermediate- risk stage IB1 cervical cancer patients
}

\author{
Asama Vanichtantikul, Patou Tantbirojn, Tarinee Manchana*
}

\begin{abstract}
Background: Survival for patients with early stage cervical cancer without any high-risk factors treated with radical hysterectomy is excellent. However, there are few data on the survival outcomes for low-risk and intermediate-risk early stage cervical cancer patients.

Objective: To determine survival outcomes and prognostic factors of low-risk and intermediate-risk stage IB1 cervical cancer patients.

Methods: Stage IB1 cervical cancer patients with radical hysterectomy and pelvic lymphadenectomy were retrospectively reviewed. Patients with positive pelvic nodes, parametrial involvement, and positive margin who are classified as high-risk patients were excluded. Patients with squamous cell carcinoma or grade 1-2 adenocarcinoma, tumor size less than $2 \mathrm{~cm}$, no lymphovascular space invasion (LVSI), and depth of stromal invasion (DSI) less than $10 \mathrm{~mm}$ were defined as low-risk patients. Survival was evaluated using the Kaplan-Meier method and compared by the log-rank test. Multivariate analysis was performed using Cox proportional-hazards regression.

Results: There were $82(42.3 \%)$ low-risk patients and $112(57.7 \%)$ intermediate-risk patients. More patients in intermediate risk received adjuvant treatment $(3.6 \%$ and $14.3 \%, P=0.07)$. Three $(3.6 \%)$ low-risk patients and $18(16.1 \%)$ intermediate-risk patients had recurrent disease $(P=0.004)$. At median follow-up of 86 months, $1.2 \%$ of low-risk patients and $8.9 \%$ of intermediate-risk had cancer-related deaths $(P=0.02)$. Low-risk patients had significantly better 5-year disease-free survival $(98.2 \%$ vs $91.1 \%, P=0.01)$ and estimated 5-year overall survival $(98.5 \%$ vs $91.1 \%$, $P=0.01$ ). DSI more than $10 \mathrm{~mm}$ and presence of LVSI were significantly associated with recurrence. However, LVSI was an independent prognostic factor.

Conclusion: Stage IB1 cervical cancer patients had excellent survival. Low-risk patients had significantly better survival. Presence of LVSI was an independent prognostic factor.
\end{abstract}

Keywords: prognostic; survival; uterine cervical neoplasms

Cervical cancer is one of the most common gynecologic malignancies and also a common cause of cancer-related death worldwide. The standard of care for patients with early stage cervical cancer is radical hysterectomy with pelvic lymph node dissection, or radiotherapy with or without concurrent chemotherapy [1]. Similar survival outcomes were reported among these modalities [2, 3]. Adjuvant radiation after radical surgery, which has been reported to decrease the incidence of local recurrence, is usually recommended for patients with an increased risk of recurrence depending on surgico-pathological

* Correspondence to: Tarinee Manchana, Department of Obstetrics and Gynecology, Faculty of Medicine, Chulalongkorn University, Bangkok 10330, Thailand, e-mail: Tarinee.M@chula.ac.th

Department of Obstetrics and Gynecology, Faculty of Medicine, Chulalongkorn University, Bangkok 10330, Thailand

כ Open Access. ๑ 2019 Asama Vanichtantikul et al., published by Sciendo. (Gc)BY-NC-ND This work is licensed under the Creative Commons Attribution NonCommercial-NoDerivatives 4.0 License. 
risk factors. High-risk factors include positive surgical margin, parametrial involvement and lymph node metastasis $[4,5]$. Intermediate-risk factors include large tumor size, lymphovascular space invasion (LVSI) and deep stromal invasion according to Sedlis criteria $[6,7]$. Patients with squamous cell carcinoma or grade 1-2 adenocarcinoma, tumor size less than 2 centimeters $(\mathrm{cm})$, no LVSI, and depth of stromal invasion (DSI) less than 10 millimeters $(\mathrm{mm})$ are classified as low-risk patients $[8,9]$.

The 5-year disease-free survival (DFS) and overall survival (OS) of patients with early stage cervical cancer treated with radical hysterectomy is approximately $74 \%-90 \%$ and $83 \%-91 \%$, respectively $[10,11]$. However, patients without high-risk factors had a higher 5-year OS (91\%-96\%) [12]. Aside from this, there are no data on the survival outcomes for low-risk and intermediate-risk early stage cervical cancer patients.

The aims of this study were to assess and compare the survival outcomes of low-risk and intermediate-risk patients with stage IB1 cervical cancer who had radical hysterectomy and pelvic lymphadenectomy and also to identify the prognostic factors associated with the survival outcomes.

\section{Materials and methods}

This study was approved by the Institutional Review Board, Faculty of Medicine, Chulalongkorn University (certificate of approval no. 866/2014). All patients provided written informed consent prior to any procedures.

This is a retrospective study that was conducted on patients with stage IB1 cervical cancer who had radical hysterectomy and pelvic lymphadenectomy between January 1997 and December 2013 at King Chulalongkorn Memorial Hospital. Patients who had incomplete or insufficient medical records, received neoadjuvant chemotherapy, lacked paraffin blocks, had grade 3 or clear cell adenocarcinoma, neuroendocrine tumors or mesenchymal tumors of histological type, had positive pelvic nodes, had parametrial involvement, and had positive margin were excluded. Clinicopathological variables such as age, parity, history of conization, tumor histology, tumor grading, tumor width, DSI, and LVSI were collected. All pathological slides were reviewed by an independent gynecologic pathologist who was blinded to the patients' outcomes. The tumor width was measured along the surface epithelium. If prior conization was performed and residual tumor was found on the hysterectomy specimen, the maximum depth of invasion was defined as the deepest invasion either on conization or hysterectomy specimen. Patients with squamous cell carcinoma or grade 1 or 2 adenocarcinoma, tumor size less than $2 \mathrm{~cm}$, no LVSI, and less than $10 \mathrm{~mm}$ of DSI were classified as low-risk patients $[8,9]$. The rest of the patients were classified as intermediate-risk patients [6-9]. DFS was defined as a period between the initial diagnosis and the time of recurrence or last follow-up. OS was defined as a period from the diagnosis to cancer-related death or last follow-up. Statistical analysis was performed using SPSS software version 22. Student $t$ test and Pearson's chi-squared test or Fisher's exact test were used to determine the differences in continuous and categorical data, respectively. Survival was evaluated using the Kaplan-Meier method and compared using the log-rank test. Multivariate analysis was performed using Cox proportionalhazards regression. Statistical significance was determined as $P$-value less than 0.05 , and the $95 \%$ confidence interval (CI) for hazard ratio was calculated.

\section{Results}

Four hundred-eleven cervical cancer patients who had radical hysterectomy and pelvic lymphadenectomy during 1997 and 2013 were identified. Eighty-two patients received neoadjuvant chemotherapy; 59 patients did not have their paraffin blocks; 30 patients had stage IA1 or IA2 on their final surgical specimen; 12 patients had clear cell adenocarcinoma, neuroendocrine tumors or mesenchymal tumors of histological type; and 34 patients had positive pelvic nodes, parametrial involvement, and positive margin; so all of them were excluded. Hence, a total of 194 patients were enrolled in this study. There were $82(42.3 \%)$ low-risk patients and $112(57.7 \%)$ intermediate-risk patients. The median age was 46 years (range 26-71 years) and was comparable between both groups (46 and 45 years, $P=0.31)$. Forty-two patients $(21.6 \%$ ) had conization prior to radical surgery. Squamous cell carcinomas were the most frequent histological type (61.3\%) detected, followed by adenocarcinoma (33\%) and adenosquamous carcinoma (5.7\%). Most of the patients (82.5\%) had microscopic lesion or tumor size less than $2 \mathrm{~cm}$. Fifty-one patients $(26.3 \%)$ had DSI more than $10 \mathrm{~mm}$ but 89 patients (45.9\%) had more than $50 \%$ of stromal invasion, and 87 patients $(44.8 \%)$ had positive LVSI.

Eighty-two patients $(42.3 \%)$ met the definition for lowrisk cervical cancer patient. Median tumor size was $1 \mathrm{~cm}$ $(0-2 \mathrm{~cm})$ and median DSI was $5.0 \mathrm{~mm}(0.5-9.9 \mathrm{~mm})$, and 65 patients $(79.3 \%)$ had less than $50 \%$ of stromal invasion. Forty-seven patients $(57.3 \%)$ had squamous cell carcinoma, and 35 patients (42.7\%) had adenocarcinoma.

Out of 112 patients with intermediate risk, the median tumor size was $1.4 \mathrm{~cm}(0-4 \mathrm{~cm})$ and median DSI was $9.1 \mathrm{~mm}$ (3-22 mm), and most of them $(64.3 \%)$ had squamous cell carcinoma. Thirty-four patients $(30.4 \%)$ had tumor size 
larger than $2 \mathrm{~cm}, 89$ patients $(79.5 \%)$ had positive LVSI, and 51 patients $(45.5 \%)$ had DSI more than $10 \mathrm{~mm}$. Sixty-two patients $(55.4 \%)$ had only 1 risk factor, 38 patients $(33.9 \%)$ had 2 risk factors, and 12 patients $(10.7 \%)$ had all the risk factors. From the intermediate-risk patients with only 1 risk factor (62 patients), majority of them (45 patients, 72.6\%) had positive LVSI, 8 (12.9\%) had large tumor size, and 9 (14.5\%) had DSI more than $10 \mathrm{~mm}$.

Nineteen patients $(9.8 \%)$ received adjuvant treatment including radiotherapy $(7.7 \%)$, chemotherapy $(0.5 \%)$ and chemoradiation $(1.5 \%)$. More patients in the intermediate-risk group had adjuvant treatment $(14.3 \%$ and $3.6 \%, P=0.07)$. Twenty-one patients $(10.8 \%)$ had recurrent disease of which 3 patients $(3.6 \%)$ were from the low-risk group and 18 patients (16.1\%) were from the intermediate-risk group $(P=0.004)$. Nineteen of the 21 patients $(90.5 \%)$ with recurrent disease did not receive any adjuvant treatment of which 3 patients were from the low-risk group and 16 patients were from the intermediate-risk group $(P=0.73)$. In contrast, only 2 of 21 patients who had received adjuvant radiation treatments $(9.5 \%)$ had recurrent disease and both of them had distant metastasis. Among the intermediate-risk patients with only 1 risk factor, 7 patients $(11.3 \%)$ had recurrent disease, 6 patients $(9.7 \%)$ had LVSI, and $1(1.6 \%)$ had a large tumor size. All of these patients did not receive adjuvant treatment. However, there was no recurrent disease among patients who had only 1 risk factor with DSI more than $10 \mathrm{~mm}$. The rate for recurrent disease was higher in patients with 2 and 3 risk factors. There were 7 patients $(18.4 \%)$ who had 2 risk factors and 4 patients $(33.3 \%)$ who had 3 risk factors. Only 1 patient in each group received adjuvant treatment.

Sub-analysis was done in 175 patients without adjuvant treatments. Patients from the intermediate-risk group had higher rates of recurrent disease $(13.5 \%$ and $3.8 \%, P=$ $0.005)$ and cancer-related deaths $(8.3 \%$ and $1.2 \%, P=0.03)$, which are shown in Table 1. Most patients (78.9\%) had either vaginal cuff or pelvic recurrence. Distant metastasis developed in 4 patients in the intermediate-risk group but none in

Table 1. Outcomes of patients without adjuvant treatment

\begin{tabular}{lccc}
\hline & $\begin{array}{c}\text { Low-risk } \\
\text { patients (n= 79) }\end{array}$ & $\begin{array}{c}\text { Intermediate-risk } \\
\text { patients (n= 96) }\end{array}$ & $\boldsymbol{P}^{*}$ \\
\hline Recurrent disease & $3(3.8 \%)$ & $13(13.5 \%)$ & 0.005 \\
Vaginal cuff & $2(2.5 \%)$ & $5(5.2 \%)$ & \\
Pelvis & $1(1.2 \%)$ & $4(4.2 \%)$ & \\
Distant & $0(0 \%)$ & $1(1 \%)$ & \\
Local and distant & $0(0 \%)$ & $3(3.1 \%)$ & \\
Cancer-related deaths & $1(1.2 \%)$ & $8(8.3 \%)$ & 0.03 \\
\hline
\end{tabular}

${ }^{*} P<0.05$ was considered as statistically significant. the low-risk group. The recurrences in the intermediate-risk group with 1-3 risk factors were $12.3 \%, 18.8 \%$, and $42.9 \%$, respectively. All of those recurrent patients with 2 and 3 risk factors had positive LVSI (93.8\%), and 7 patients (43.8\%) had more than $10 \mathrm{~mm}$ stromal invasion. The cancer-related deaths occurred in 8 patients after recurrence (50\%), of which 5 were pelvic recurrence and 3 were distant recurrence. The median follow-up was 86 months (range 1-215 months). The cancer-related deaths occurred in 11 patients $(5.7 \%)$, of which 1 was from the low-risk group $(1.2 \%)$ and 10 were from the intermediate-risk group $(8.9 \%)(P=0.02)$. Nine of these patients $(81.8 \%), 1$ patient from the low-risk group and 8 patients from the intermediate-risk group, did not receive adjuvant treatment and developed recurrent disease.

There was a significant difference in DFS between both groups as shown in Figure $1(98.2 \%$ vs $91.1 \%, P=0.01)$. The estimated 5-year OS was also statistically significant (98.5\% vs $91.1 \%, P=0.01$ ), which is shown in Figure 2. DSI more than $10 \mathrm{~mm}$ and LVSI were significant variables associated with a worse DFS. Tumor size, histological type, percentage of stromal invasion, and adjuvant therapy were not significant prognostic factors as shown in Table 2. Multivariate analysis demonstrated that only LVSI remained statistically significant with hazard ratio of $5.84(95 \% \mathrm{CI}, 1.1$ to $28.1, P=0.03)$.

\section{Discussion}

The results from this study corroborate the findings previously reported [10-12] that stage IB1 cervical cancer patients treated with radical surgery had good survival outcomes. However, when the survival outcomes of low-risk patients were compared to those of intermediate-risk patients, there were significant differences detected. Previous studies [6, 7] showed that the presence of 3 intermediate-risk factors were associated with poor survival outcomes. This study confirmed that the survival outcomes of low-risk patients were significantly better than those of intermediate-risk patients.

The favorable histological type, tumor size less than $2 \mathrm{~cm}$, DSI less than $10 \mathrm{~mm}$, and no LVSI had been reported to have better prognosis. For this study, the squamous cell carcinoma was the most common histological type detected, which was similar to previous studies $[6,10,11]$. However, the prognostic significance of histology is quite controversial. Some studies showed adenocarcinoma as a poor prognostic factor, whereas others have reported that it could not distinguish between adenocarcinoma and squamous cell carcinoma [13-15]. For this study, the histological type was not an independent prognostic factor. Other prognostic factors for early stage cervical cancer have been reported such as tumor size, LVSI, and DSI [7]. 


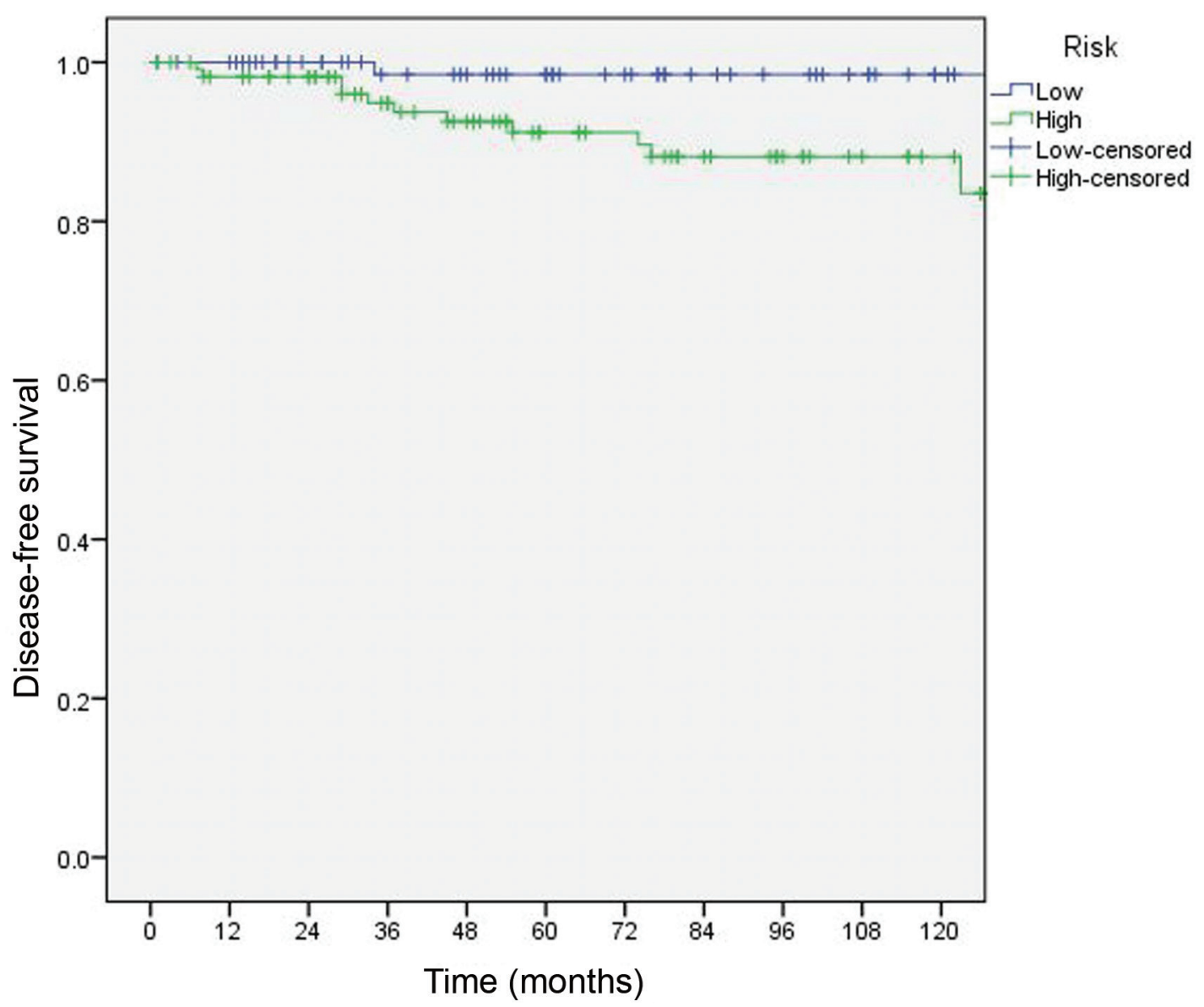

Figure 1. Disease-free survival between low-risk and intermediate-risk stage IB cervical cancer patients

Because of these factors, the authors evaluated the survival outcomes of 194 cervical cancer stage IB1 patients with and without these factors. DSI more than $10 \mathrm{~mm}$ and LVSI were significantly associated with a worse DFS. However, after multivariate analysis, LVSI remained as an independent prognostic factor. This result was comparable to previous studies that showed that LVSI was a poor prognostic factor $[7,10$, 16-18]. Twenty percent of the patients with LVSI had recurrent disease, but only $4 \%$ of the patients without LVSI had recurrent disease. Therefore, LVSI alone may be sufficient enough to provide information whether the patient should have an adjuvant treatment.

DSI is also an important prognostic factor. However, there are no consensuses for the cutoff level. Traditionally, more than one-third stromal invasion was used for 1 intermediate risk [6]. Unfortunately, more than $50 \%$ of stromal invasion is usually defined as having deep stromal invasion, and it was found to be as an independent risk factor for recurrence [19, 20]. Nevertheless, the findings from this study support that DSI more than $10 \mathrm{~mm}$ was associated with a poorer survival outcome [18, 21].

As for the tumor size, many studies revealed that size more than $4 \mathrm{~cm}$ can affect the survival outcomes [7, 10, 11].
Sevin et al. showed that tumor size larger than $2 \mathrm{~cm}$ was a significant prognostic factor [18]. In contrast, this study did not detect any significant differences among patients with tumor size larger than $2 \mathrm{~cm}$. Since a large tumor size often associated with parametrial and pelvic node involvement [18, 22], however patients with parametrial or pelvic node involvement were excluded from this study. This may explain why the tumor size alone was not associated with poorer outcome in this study.

The GOG study had established that certain patients with intermediate risk had a $31 \%$ recurrent risk after radical hysterectomy and pelvic lymphadenectomy [7]. Intermediate-risk patients in the GOG study were defined as those who had at least 2 out of 3 risk factors; however, the intermediate-risk patients in this study had at least 1 risk factor. As a result of this, the recurrent rate was lower [16.1\%] in this study. Regardless of these findings, it has been reported that adjuvant radiotherapy can significantly lower the recurrent rate in women with these risk factors [6]. The results from this study corroborated that few patients with adjuvant treatment had recurrent disease. However, it should also be noted that the adjuvant treatment cannot improve the DFS because the 


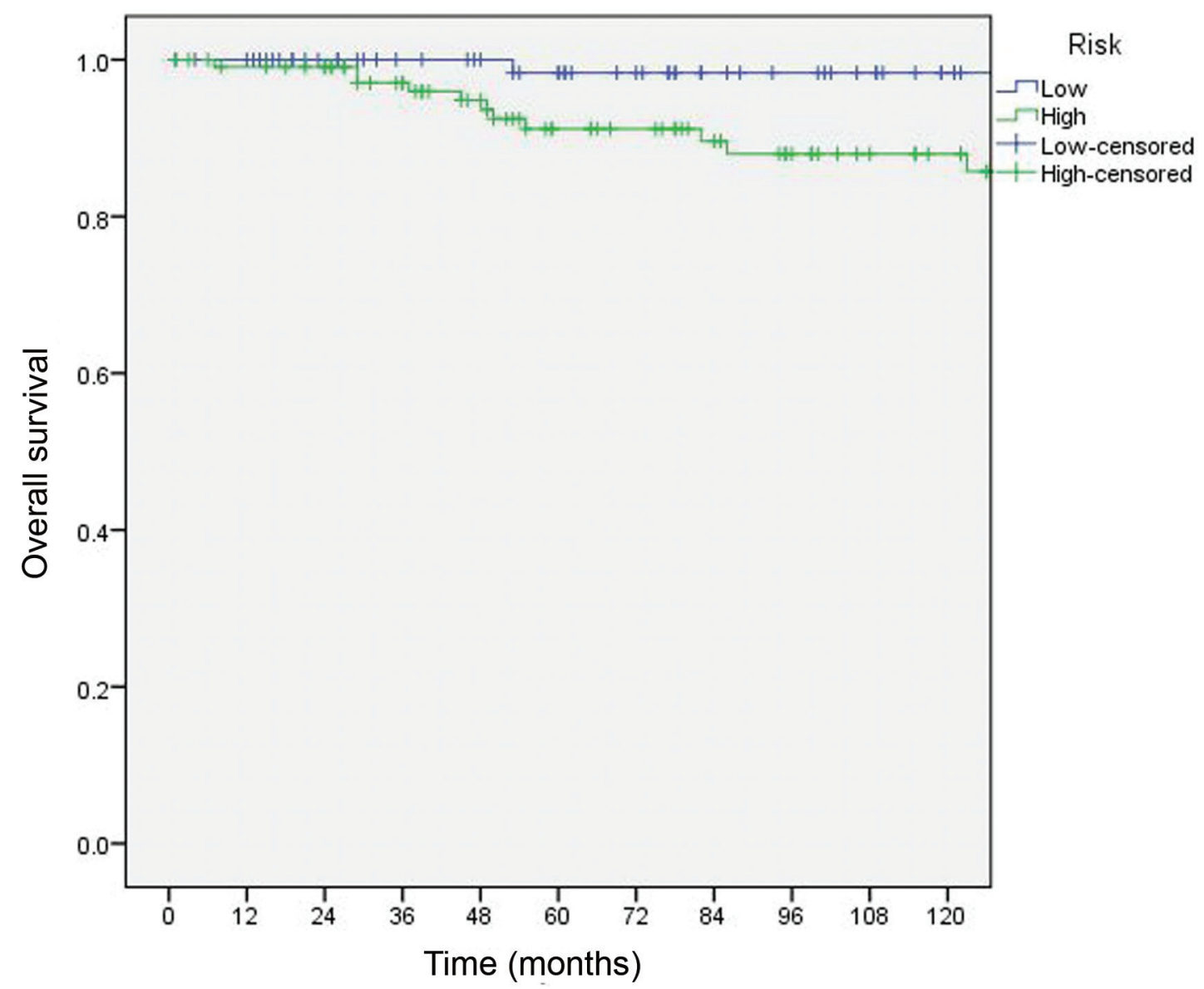

Figure 2. Overall survival between low-risk and intermediate-risk stage IB cervical cancer patients

Table 2. Prognostic variables associated with a 5-year DFS

\begin{tabular}{lccc}
\hline Variables & 5-year DFS (\%) & $\boldsymbol{P}$ & $\mathbf{9 5 \%} \mathbf{C l}$ \\
\hline Histology & & 0.79 & $0.35-2.33$ \\
$\quad$ Squamous cell carcinoma & 92.7 & & \\
$\quad$ Adenocarcinoma & 98.2 & & \\
$\quad$ Adenosquamous carcinoma & 83.4 & & \\
Depth of stromal invasion & & 0.04 & $1.02-9.00$ \\
$\quad$ 10 millimeters or less & 95.2 & & \\
$\quad$ More than 10 millimeters & 91.1 & & \\
Stromal invasion (\%) & & 0.27 & $0.60-5.66$ \\
$\quad<50 \%$ & 94.9 & & \\
$\quad>50 \%$ & 93.6 & & \\
$\quad$ Tumor size & & 0.12 & $0.75-7.97$ \\
$\quad$ centimeters or less & 94.1 & & \\
$\quad$ More than 2 centimeters & 96.6 & & \\
Lymphovascular space invasion & & 0.003 & $1.59-32.32$ \\
$\quad$ Negative & 97.4 & & \\
$\quad$ Positive & 90 & & \\
Adjuvant therapy & & 0.23 & $0.44-3.87$ \\
$\quad$ No & 94.6 & & \\
$\quad$ Yes & 99.8 & & \\
\hline
\end{tabular}

$\mathrm{Cl}$, confidence interval; DFS, disease-free survival. number of recurrent patients in the intermediate-risk group was too small to detect any significant differences. Even though adjuvant radiotherapy can lower the recurrent rate, but its morbidities related to the radiation such as bladder and bowel dysfunction are of concern [23, 24]. As a result of this, adjuvant radiotherapy should be reserved for patients with high risk for recurrent disease. Adjuvant radiotherapy is not recommended for patients with low risk for recurrent disease, and those patients with only 1 risk factor, besides LVSI, did not benefit from such treatment. Considering this, LVSI is an important prognostic factor that is independently associated with the survival outcome.

\section{Conclusion}

Stage IB1 cervical cancer patients who had radical surgery generally had excellent survival outcome, but low-risk patients had a better survival outcome compared to intermediaterisk patients. LVSI was independently associated with a better survival outcome. Thus, an adjuvant treatment may be beneficial for patients with LVSI. 
Author contributions. All the authors contributed substantially to the concept and design of this study. AV and PT contributed substantially to the acquisition of data. PT and TM analyzed and interpreted the data. AV drafted the manuscript. PT and TM contributed substantially to its critical revision. All the authors approved the final version submitted for publication and take responsibility for the statements made in the published article.

Acknowledgments. The authors thank the staff of Division of Gynecologic Oncology and Cyto-pathology, Department of Obstetrics and Gynecology, Faculty of Medicine, Chulalongkorn University, for their support in the data collection.

Conflict of interest statement. The authors have completed and submitted the International Committee of Medical Journal Editors Uniform Disclosure Form for Potential Conflicts of Interest. None of the authors disclose any conflict of interest.

\section{References}

[1] Landoni F, Maneo A, Colombo A, Placa F, Milani R, Perego P, et al. Randomised study of radical surgery versus radiotherapy for stage Ib-IIa cervical cancer. Lancet. 1997; 350:535-40.

[2] Delgado G, Bundy BN, Fowler WC Jr., Stehman FB, Sevin B, Creasman WT, et al. A prospective surgical pathological study of stage I squamous carcinoma of the cervix: a Gynecologic Oncology Group Study. Gynecol Oncol. 1989; 35:314-20.

[3] Landoni F, Maneo A, Cormio G, Perego P, Milani R, Caruso O, et al. Class II versus class III radical hysterectomy in stage IB-IIA cervical cancer: a prospective randomized study. Gynecol Oncol. 2001; 80:3-12.

[4] Kinney WK, Alvarez RD, Reid GC, Schray MF, Soong SJ, Morley GW, et al. Value of adjuvant whole-pelvis irradiation after Wertheim hysterectomy for early-stage squamous carcinoma of the cervix with pelvic nodal metastasis: a matched-control study. Gynecol Oncol. 1989; 34:258-62.

[5] Peters WA 3rd, Liu PY, Barrett RJ 2nd, Stock RJ, Monk BJ, Berek JS, et al. Concurrent chemotherapy and pelvic radiation therapy compared with pelvic radiation therapy alone as adjuvant therapy after radical surgery in high-risk early-stage cancer of the cervix. J Clin Oncol. 2000; 18:1606-13.

[6] Sedlis A, Bundy BN, Rotman MZ, Lentz SS, Muderspach LI, Zaino RJ. A randomized trial of pelvic radiation therapy versus no further therapy in selected patients with stage IB carcinoma of the cervix after radical hysterectomy and pelvic lymphadenectomy: a Gynecologic Oncology Group Study. Gynecol Oncol. 1999; 73:177-83.

[7] Delgado G, Bundy B, Zaino R, Sevin BU, Creasman WT, Major F. Prospective surgical-pathological study of disease-free interval in patients with stage IB squamous cell carcinoma of the cervix: a Gynecologic Oncology Group study. Gynecol Oncol. 1990; 38:352-7.

[8] Covens A, Rosen B, Murphy J, Laframboise S, DePetrillo AD, Lickrish $\mathrm{G}$, et al. How important is removal of the parametrium at surgery for carcinoma of the cervix? Gynecol Oncol. 2002; 84:145-9.
[9] Stegeman M, Louwen M, van der Velden J, ten Kate FJ, den Bakker MA, Burger CW, et al. The incidence of parametrial tumor involvement in select patients with early cervix cancer is too low to justify parametrectomy. Gynecol Oncol. 2007; 105:475-80.

[10] Ayhan A, Al RA, Baykal C, Demirtas E, Yuce K, Ayhan A. A comparison of prognoses of FIGO stage IB adenocarcinoma and squamous cell carcinoma. Int J Gynecol Cancer. 2004; 14:279-85.

[11] Trimbos JB, Lambeek AF, Peters AA, Wolterbeek R, Gaarenstroom KN, Fleuren GJ, et al. Prognostic difference of surgical treatment of exophytic versus barrel-shaped bulky cervical cancer. Gynecol Oncol. 2004; 95:77-81.

[12] Singh P, Tripcony L, Nicklin J. Analysis of prognostic variables, development of predictive models, and stratification of risk groups in surgically treated FIGO early-stage (IA-IIA) carcinoma cervix. Int J Gynecol Cancer. 2012; 22:115-22.

[13] Burke TW, Hoskins WJ, Heller PB, Bibro MC, Weiser EB, Park RC. Prognostic factors associated with radical hysterectomy failure. Gynecol Oncol. 1987; 26:153-9.

[14] Nakanishi T, Ishikawa H, Suzuki Y, Inoue T, Nakamura S, Kuzuya K. A comparison of prognoses of pathologic stage Ib adenocarcinoma and squamous cell carcinoma of the uterine cervix. Gynecol Oncol. 2000; 79:289-93.

[15] Shingleton HM, Bell MC, Fremgen A, Chmiel JS, Russell $\mathrm{AH}$, Jones $\mathrm{WB}$, et al. Is there really a difference in survival of women with squamous cell carcinoma, adenocarcinoma, and adenosquamous cell carcinoma of the cervix? Cancer. 1995; 76 Suppl 10:1948-55.

[16] Ishikawa H, Nakanishi T, Inoue T, Kuzuya K. Prognostic factors of adenocarcinoma of the uterine cervix. Gynecol Oncol. 1999; 73:42-6.

[17] Matthews CM, Burke TW, Tornos C, Eifel PJ, Atkinson EN, Stringer CA, et al. Stage I cervical adenocarcinoma: prognostic evaluation of surgically treated patients. Gynecol Oncol. 1993; 49:19-23.

[18] Sevin BU, Nadji M, Lampe B, Lu Y, Hilsenbeck S, Koechli OR, et al Prognostic factors of early stage cervical cancer treated by radical hysterectomy. Cancer. 1995; 76 Suppl 10:1978-86.

[19] Lee KB, Lee JM, Ki KD, Lee SK, Park CY, Ha SY. Comparison of adjuvant chemotherapy and radiation in patients with intermediate risk factors after radical surgery in FIGO stage IB-IIA cervical cancer. Int J Gynecol Cancer. 2008; 18:1027-31.

[20] Takeshima N, Umayahara K, Fujiwara K, Hirai Y, Takizawa K, Hasumi K. Treatment results of adjuvant chemotherapy after radical hysterectomy for intermediate- and high-risk stage IB-IIA cervical cancer. Gynecol Oncol. 2006; 103:618-22.

[21] Boyce J, Fruchter RG, Nicastri AD, Ambiavagar PC, Reinis MS, Nelson JH Jr. Prognostic factors in stage I Carcinoma of the cervix. Gynecol Oncol. 1981; 12:154-65.

[22] Gemer O, Eitan R, Gdalevich M, Mamanov A, Piura B, Rabinovich A, et al. Can parametrectomy be avoided in early cervical cancer? An algorithm for the identification of patients at low risk for parametrial involvement. Eur J Surg Oncol. 2013; 39:76-80.

[23] Manchana T, Sirisabya N, Lertkhachonsuk R, Worasethsin P, Khemapech N, Sittisomwong T, et al. Long term complications after radical hysterectomy with pelvic lymphadenectomy. J Med Assoc Thai. 2009; 92:451-6.

[24] Ralph G, Winter R, Michelitsch L, Tamussino K. Radicality of parametrial resection and dysfunction of the lower urinary tract after radical hysterectomy. Eur J Gynaecol Oncol. 1991; 12:27-30. 